\title{
Deployment Optimization of Small Cell Networks with Sleep Mode
}

DOI:

10.1109/TVT.2019.2935403

\section{Document Version}

Accepted author manuscript

Link to publication record in Manchester Research Explorer

\section{Citation for published version (APA):}

Mugume, E., \& So, D. K. C. (2019). Deployment Optimization of Small Cell Networks with Sleep Mode. IEEE Transactions on Vehicular Technology. https://doi.org/10.1109/TVT.2019.2935403

\section{Published in:}

IEEE Transactions on Vehicular Technology

\section{Citing this paper}

Please note that where the full-text provided on Manchester Research Explorer is the Author Accepted Manuscript or Proof version this may differ from the final Published version. If citing, it is advised that you check and use the publisher's definitive version.

\section{General rights}

Copyright and moral rights for the publications made accessible in the Research Explorer are retained by the authors and/or other copyright owners and it is a condition of accessing publications that users recognise and abide by the legal requirements associated with these rights.

\section{Takedown policy}

If you believe that this document breaches copyright please refer to the University of Manchester's Takedown Procedures [http://man.ac.uk/04Y6Bo] or contact uml.scholarlycommunications@manchester.ac.uk providing relevant details, so we can investigate your claim.

\section{OPEN ACCESS}




\title{
Deployment Optimization of Small Cell Networks with Sleep Mode
}

\author{
Edwin Mugume ${ }^{\dagger}$, Member, IEEE, and Daniel K. C. So ${ }^{\ddagger}$, Senior Member, IEEE
}

\begin{abstract}
Demand for mobile data services is increasing exponentially and operators have responded by expanding and upgrading their networks to enhance capacity. One promising technique is to densify the network with low-power and smallcoverage base stations (BSs) to provide significant capacity gains. In this paper, we develop a multi-user connectivity model for a Poisson Point Process (PPP)-based homogeneous network to facilitate a realistic study of its energy performance subject to blocking constraints. We then derive the average rate per channel and per user and compute the average sum rate of the network. An area power consumption (APC)-minimization framework is then formulated subject to appropriate coverage and average rate constraints to determine the optimal deployment configuration of BS density and associated transmit power. Furthermore, a new sleep mode mechanism called centralized strategic scheme is analyzed to determine its ability to adapt energy consumption to variable user density. Our results show that sleep mode is a viable solution for managing network energy consumption in dense networks.
\end{abstract}

Index Terms-Homogeneous network, optimization, area power consumption, sleep mode, energy efficiency.

\section{INTRODUCTION}

$\mathbf{M}$ OBILE network operators are faced with exponentiallyincreasing data traffic demand caused by significant advances in mobile cellular technologies, smart end-user devices and their associated data-hungry applications [1]-[2]. A Cisco forecast of the global mobile data traffic for the period 2016-2021 shows a compound annual growth rate of 47\% [2], which is a major challenge for current systems and future $5 \mathrm{G}$ networks [3]-[4]. However, the capacities of individual links in current network designs are close to their theoretical limits. Macro base stations (BSs) which dominate current networks consume a lot of energy and this has raised economic and environmental concerns over increasing energy cost and the associated greenhouse gas emissions. A popular strategy to address these challenges is to reduce the average size of cells which enhances frequency reuse and bandwidth per user [5]. Other techniques to enhance network capacity include deployment of heterogeneous cellular networks (HetNets) [6]-

$\dagger^{\dagger}$. Mugume was previously with the Department of Electrical and Electronic Engineering, The University of Manchester, in Manchester, United Kingdom. He is now with Carnegie Mellon University Africa in Kigali, Rwanda, and Makerere University in Kampala, Uganda (email: emugume@andrew.cmu.edu).

$\ddagger_{D}$. K. C. So is with the School of Electrical and Electronic Engineering, The University of Manchester, Manchester, M13 9PL, United Kingdom (email: d.so@manchester.ac.uk).

Copyright (c) 2015 IEEE. Personal use of this material is permitted. However, permission to use this material for any other purposes must be obtained from the IEEE by sending a request to pubs-permissions@ieee.org.
[7], MIMO and massive MIMO techniques [8]-[9], cognitive radio systems [10], among others.

A dense deployment of small BSs can bring significant coverage and capacity gains to mobile users. However, even though small BSs consume much less power than macro BSs [11], their deployment in large numbers may worsen the aggregate power consumption. It is well known that traffic intensity highly varies spatiotemporally throughout each 24hour day, peaking during the day and early evening hours [12]. However, cellular networks are traditionally designed to support peak traffic at all times, meaning that a large proportion of network resources may become idle when traffic demand inevitably drops. Idle BSs still consume significant power which negatively impacts energy performance [11], [13]. It is therefore important to adapt power consumption to variations in the network load.

Our paper combines two specific ideas of deployment strategy and sleep mode mechanisms to enhance network energy performance. Some works have focused on network deployment strategies [14]-[16]. Authors in [14] show that the energy efficiency (EE) of a homogeneous network can be enhanced by densifying it with more BSs coupled with reduced transmit power. Authors in [15] jointly optimize the BS densities and transmit powers per tier in a two-tier HetNet to minimize the area power consumption (APC) subject to a coverage probability constraint. In [16], the BS density and transmit power are jointly optimized to minimize the APC subject to coverage and rate constraints under different network conditions. However, these works do not describe how to choose the BSs that remain active as conditions change.

Other works have focused on managing network power consumption using sleep mode mechanisms [17]-[24]. Sleep mode decisions should consider the load profile in the wider geographical coverage of the network as opposed to localized decisions [17]. Furthermore, network coverage and energy performance are improved when cells are switched off based on their levels of activity [18]. Authors in [19] study an energy minimization problem subject to downlink coverage and uplink power constraints. Some macro BSs are switched off and replaced by micro BSs coupled with power adjustment techniques. In [20], a joint optimization of BS sleeping control and power matching schemes is performed to achieve flexible tradeoffs between power consumption and quality of service (QoS). In [21], a technique is proposed to manage power consumption by switching off some BSs and balancing the prevailing traffic load between the remaining active BSs. Our work in [22] studied a sleep mode strategy which prioritizes cells with the fewest users for sleep mode; results showed 
enhanced network coverage, average rate and EE. In [23]-[24], authors considered dynamic long-term traffic and jointly optimized cell association, cell activation, and spectrum allocation in their study of energy aware management in HetNets.

The time domain operation of sleep mode has been studied extensively in literature with the objective of ensuring that BS switch on/off strategy is not too disruptive but can still quickly respond to new traffic requests. Some approaches, for instance [25], consider two stages of sleep mode i.e. light and deep sleep stages. The deep sleep strategy is influenced by slow-varying long-term traffic and can therefore be used to determine how many BSs to switch off for a given time interval. During such an interval, all remaining BSs only save energy using the light sleep strategy and are able to quickly wake up and serve new users. In another approach, [26] combines deep sleep strategies for long-term traffic variation and discontinuous transmission which implements micro-sleep schemes for short-term traffic variations.

In the stochastic geometry approach, some works such as [18], [27] assume that each cell randomly selects and connects one user in its coverage area but this is unrealistic from an EE point of view if the network is subjected to blocking constraints. Blocking performance is especially important in a network implementing sleep mode strategies to guarantee QoS [28]. Other works assume that the user density is so large that each cell has at least one user and therefore always transmits [16], [27]. This is also generally unrealistic since the user density is finite and spatiotemporally variable. Our work relaxes these assumptions to obtain a more generic and realistic analysis of the performance of the network.

In general, locations of small BSs are highly irregular and there is a high degree of randomness of cell sizes and shapes. Hence, grid-based cellular topologies are too idealized to effectively study dense small cell networks. Random spatial techniques that model both BS and user locations as independent Poisson Point Processes (PPPs) have been proposed in recent years [29]-[30]. The approach has been applied to study homogeneous networks [27], HetNets [6]-[7], cognitive radio systems [10], network optimization [14], [19], [31], etc. It facilitates simple and tractable network analysis and often provides closed-form expressions. Moreover, it has been shown to provide a tight approximation of the performance of practical networks [27], [32], [33]. We therefore use this approach for the analysis in this paper.

\section{A. Main Contributions and Outcomes}

In this paper, we first develop a multi-user connectivity model that facilitates connection of multiple users at each BS. We then study sleep mode techniques that can utilize this model to adapt energy consumption to inevitable variations in user density. The study is based on a homogeneous small cell network where some cells may be idle (have no users) while others contain more users than the available physical channels. We derive the average rate per channel, average user rate and average sum rate of the network using simple channel allocation assumptions. This paper significantly extends our previous work in [22] which only considers conventional sleep mode where idle BSs are put into sleep mode. Our work also uses and builds upon some of the performance analysis of a homogeneous network shown in [27]. However, our major contributions are significantly different and can be summarized as follows:

- Our analysis relaxes the 'single-channel network' assumption in [27] to analyze a more realistic multi-channel network in which each BS has the ability to connect multiple users. This multi-user connectivity model is also very important in a scenario where network analysis is subjected to blocking or connectivity constraints.

- We formulate an APC-minimization framework to determine the optimal combination of BS density and transmit power per BS subject to coverage probability and average rate constraints. This analysis considers the effect of idle and sleep mode BSs to derive more realistic results.

- In addition, we propose a new centralized strategic sleep mode scheme that considers the long-term spatiotemporal distribution and variation of traffic to minimize disruptions due to sleep mode and enhance network performance. The multi-user connectivity model facilitates analysis by enabling affected users to connect to other suitable active BSs.

- To simplify implementation in the network, a distributed version of the strategic sleep mode algorithm is analyzed. The distributed strategic sleep mode scheme shows a slight degradation of performance compared to its centralized counterpart.

The results of this analysis show that the criteria of choosing BSs for sleep mode is a major determinant of the resulting coverage probability, average rate and energy performance of the network. Our proposed strategic sleep mode schemes enhance the average rate of each remaining active BS which improves network resource utilization.

\section{B. Paper Organization}

The rest of the paper is organized as follows. Section II discusses the system model and assumptions. Section III models single user and multi-user connectivity at the BS. Section IV presents a constrained APC-minimization framework using only conventional sleep mode. Section V proposes and discusses our proposed sleep mode strategies. Section VI presents the numerical results and discussions. The paper is concluded in Section VII.

\section{SySTEM MODEL}

\section{A. Network Topology}

Consider a PPP-based homogeneous small cell network in which both BSs and users are located according to independent homogeneous PPPs $\Phi_{b}$ of intensity $\lambda_{b}$ and $\Phi_{u}$ of intensity $\lambda_{u}$ respectively in the $\mathbb{R}^{2}$ plane [27]. Shadowing effects are ignored and universal frequency reuse is considered in the analysis. We consider a total network area $\mathcal{A}=x^{2} \mathrm{~m}^{2}$ and assume that all BSs transmit power $P_{t}$. Hence, each user connects to its nearest BS which makes the cellular layout resemble the Poisson Voronoi (PV) tessellation [27]. Table I shows a summary of the common notations used in this paper. 
TABLE I

SUMMARY OF NOTATIONS

\begin{tabular}{|c|l|}
\hline \hline Variable & Description of variable \\
\hline$\alpha$ & Pathloss exponent with a value in range $(2,4]$ \\
\hline$\sigma^{2}$ & Additive noise power \\
\hline $\mathcal{K}$ & A constant equal to 3.575 \\
\hline $\mathcal{B}, \delta$ & System bandwidth, and number of channels \\
\hline$A ; \mathcal{A}$ & Area of a cell; Total network area \\
\hline$L$ & Pathloss constant \\
\hline$N_{t x}, P_{0}, \Delta$ & BS power consumption parameters \\
\hline$\lambda_{b} ; \lambda_{u}$ & BS density; User density \\
\hline$p_{a}$ & Probability of a BS being active \\
\hline $\bar{p}_{a}$ & Proportion of interfering BSs i.e. $\bar{p}_{a} \lambda_{b}=p_{a} \lambda_{b} \backslash b_{o}$ \\
\hline$p_{r}$ & Random thinning probability in random sleep mode \\
\hline$p_{s}$ & $\begin{array}{l}\text { Proportion of BSs available for user association in } \\
\text { centralized sleep mode }\end{array}$ \\
\hline
\end{tabular}

\section{B. Channel Model}

Consider a typical user located at the origin, a distance of $r$ from its parent BS $b_{o}$. The considered pathloss model is $l(r)=L\|r\|^{-\alpha}$, where $L$ is a constant and $\alpha>2$ is the pathloss exponent. The power received by the typical user from its parent BS is $P_{r}(r)=P_{t} h L r^{-\alpha}$, where $h$ models the fading loss between the typical user and its parent BS. After cell association, any idle BSs will thin the aggregate interference suffered by the typical user. Denoting the set of idle BSs as $\left\{b_{i d}\right\}$, the SINR of the typical user is expressed as

$$
\operatorname{SINR}=\frac{h r^{-\alpha}}{\frac{\sigma^{2}}{P_{t} L}+\sum_{i \in \Phi_{b} \backslash\left\{b_{o} \cup b_{i d}\right\}} g_{i} R_{i}^{-\alpha}}
$$

where $\sigma^{2}$ is additive noise power, $g_{i}$ is the fading loss between the typical user and the $i$-th interfering $\mathrm{BS}$ and $R_{i}$ is the distance between the typical user and the $i$-th interfering BS. Both $h$ and $g_{i}$ are assumed to be i.i.d. exponential where $h, g_{i} \sim \exp (1)$.

\section{Base Station Power Model}

A BS consumes different amounts of power depending on its operating mode. In active mode, the power consumed by a BS, denoted as $P_{A c t}$, constitutes contributions from the power amplifier, signal processing, cooling, antenna and feeder losses, etc. In light sleep mode, a BS consumes less power, denoted as $P_{\text {Lsleep }}$. When the BS is switched off (denoted as deep sleep mode in this paper), it consumes no power i.e. $P_{\text {Dsleep }}=0$. Hence, the power consumed by a typical BS can be expressed as [11]:

$P_{\text {in }}: \begin{cases}P_{\text {Act }}=N_{t x} P_{0}+\Delta P_{t}, & 0<P_{t} \leq \bar{P}_{t} \text { (Active mode) } \\ P_{\text {Lsleep }}=N_{t x} P_{s l}, & P_{t}=0 \text { (Light sleep mode) } \\ P_{\text {Dsleep }}=0, & \text { Deep sleep mode },\end{cases}$

where $N_{t x}$ is the number of transceiver chains, $P_{0}$ is the fixed no-load power consumption, $\Delta$ is the slope of the loaddependent power consumption, $\bar{P}_{t}$ is the maximum transmit power, and $P_{s l}$ is the sleep mode power consumption per transceiver chain. We use APC and EE to evaluate energy performance. The APC in Watts $/ \mathrm{m}^{2}$ is calculated as

$$
\mathrm{APC}=\lambda_{b}\left[p_{a}\left(P_{\text {Act }}-P_{\text {Lsleep }}\right)+P_{\text {Lsleep }}\right],
$$

where $p_{a}$ is the fraction of all $\mathrm{BSs}$ that are active. In contrast, EE depends on the network sum rate which is a sum of all the capacity achieved by all users in the network. EE quantifies the number of bits transmitted per unit power consumption as

$$
\mathrm{EE}=\frac{\text { Network sum rate }}{\lambda_{b} \mathcal{A}\left[p_{a}\left(P_{\text {Act }}-P_{\text {Lsleep }}\right)+P_{\text {Lsleep }}\right]} \quad[\text { bits/Joule }] .
$$

Network sum rate depends on the sleep mode strategy and will be analyzed later in the paper.

\section{Network Performance Analysis}

In a 2-D PV tessellation, the distribution of cell sizes can be approximated as [22], [34]

$$
f_{X}(A)=\frac{\left(\mathcal{K} \lambda_{b}\right)^{\mathcal{K}}}{\Gamma(\mathcal{K})} A^{\mathcal{K}-1} e^{-\mathcal{K} \lambda_{b} A}
$$

where $X$ is the cell size random variable, $\mathcal{K}=3.575$ is a constant and $\Gamma(t)=\int_{0}^{\infty} x^{t-1} e^{-x} \mathrm{~d} x$ is the gamma function. The number of users in a typical cell of area $A$ is Poisson distributed as $g_{A}(n)=\frac{\left(\lambda_{u} A\right)^{n}}{n !} e^{-\lambda_{u} A}$. Hence, the probability that a typical cell contains $n$ users is expressed as

$$
\begin{aligned}
\mathbb{P}(N=n) & =\int_{0}^{\infty} \mathbb{P}[N=n \mid X=A] \cdot f_{X}(A) \mathrm{d} A \\
& \stackrel{(a)}{=} \frac{\lambda_{u}^{n}\left(\mathcal{K} \lambda_{b}\right)^{\mathcal{K}}}{\Gamma(\mathcal{K}) n !} \int_{0}^{\infty} A^{n+\mathcal{K}-1} e^{-\left(\lambda_{u}+\mathcal{K} \lambda_{b}\right) A} \mathrm{~d} A \\
& =\frac{\lambda_{u}{ }^{n}\left(\mathcal{K} \lambda_{b}\right)^{\mathcal{K}} \Gamma(n+\mathcal{K})}{\Gamma(\mathcal{K}) n !\left(\lambda_{u}+\mathcal{K} \lambda_{b}\right)^{n+\mathcal{K}}},
\end{aligned}
$$

where $(a)$ is solved using the identity [35, (3.381.4)].

Due to the independent distribution of BSs and users, some cells may be idle (have no users). Hence, the probability of a typical BS being active, denoted as $p_{a}$, is expressed as

$$
p_{a}=1-\mathbb{P}(N=0)=1-\left(1+\frac{\lambda_{u}}{\mathcal{K} \lambda_{b}}\right)^{-\mathcal{K}} .
$$

It is assumed that in each active BS, all downlink channels are occupied which is a reasonable assumption for a homogeneous network in a high density environment such as an urban area. If any idle BSs exist in the network (i.e. $p_{a}<1$ ), then aggregate interference reduces since idle BSs do not transmit. Slivnyak's theorem, which states that an independently thinned PPP is also a PPP [30], is applied in subsequent analysis.

\section{A. Average Rate Performance}

1) Single User Connectivity Model: Some works assume that each cell connects only one user who utilizes the whole band; for instance [18], [27]. We denote this as the single user connectivity model. In a cell with multiple users, one user is chosen randomly and served by the BS. In other words, the average number of served users is equal to the average number of active BSs. Hence such a model requires a very high BS density to achieve an acceptable blocking rate. Consider a new measure called average connectivity ratio (ACR) which is introduced in [22] and expresses the proportion of all users that get a connection. It is expressed as [22]

$$
\operatorname{ACR}(v)=\frac{p_{a} \lambda_{b}}{\lambda_{u}}=\frac{\lambda_{b}}{\lambda_{u}}\left[1-\left(1+\frac{\lambda_{u}}{\mathcal{K} \lambda_{b}}\right)^{-\mathcal{K}}\right],
$$


where $v=\lambda_{b} / \lambda_{u}$ is the BS-user density ratio. To guarantee that a given blocking constraint $\chi$ is always achieved, the minimum BS density is determined as

$$
\operatorname{ACR}(v) \equiv \frac{p_{a} \lambda_{b}}{\lambda_{u}} \geq 1-\chi ; \quad \Rightarrow \quad p_{a} \lambda_{b} \geq(1-\chi) \lambda_{u}
$$

It is not possible to express (9) in closed form as a function of $\lambda_{b}$. However, $\chi$ is always set as a small number in practice. For further analysis, we approximate $p_{a}$ using the first three terms of its binomial series as

$$
p_{a} \approx \frac{\lambda_{u}}{\lambda_{b}}\left[1-\frac{\lambda_{u}}{\lambda_{b}}\left(\frac{\mathcal{K}+1}{2 \mathcal{K}}\right)\right]
$$

Thus, the required minimum BS density in closed form is expressed as

$$
\lambda_{b}=\left(\frac{\mathcal{K}+1}{2 \mathcal{K} \chi}\right) \lambda_{u}
$$

As expected therefore, $\lambda_{b}$ increases with $\lambda_{u}$ but reduces when the blocking constraint is relaxed. The exact value of $\lambda_{b}$ in (9) can be obtained using the bisection method [36].

To illustrate, consider $\chi=0.1, \lambda_{u}=10^{-4} \mathrm{~m}^{-2}$ and $\mathcal{A}=2.5 \mathrm{~km} \times 2.5 \mathrm{~km}$ such that $\lambda_{b} \geq 6.4 \times 10^{-4} \mathrm{~m}^{-2}$. Then, the average number of users and BSs are $\lambda_{u}|\mathcal{A}|=625$ and $\lambda_{b}|\mathcal{A}|=4000$ respectively. In this example, the exact BS density is obtained using (9) as $\lambda_{b}=6.98 \times 10^{-4} \mathrm{~m}^{-2}$; hence, this approximation gives a $9 \%$ difference but it allows a closed form result. It is clear that the average number of required BSs far outweighs the average number of connected users which is unrealistic in practice.

The single-user connectivity approach has various limitations especially with regard to energy consumption and efficiency. It works under the assumption that the user density is sufficiently high such that all BSs cover at least one user; [27] uses this assumption. Then, average sum rate is determined by multiplying the average user rate and the number of connected users (equivalent to the number of BSs). In a realistic network however, user connectivity is influenced by the user and BS densities and their spatial distribution. Since some BSs remain idle while some users remain unconnected, sum rate becomes difficult to determine under this approach. It becomes necessary to develop a general user connectivity framework that works under all possible combinations of BSs and users to derive an accurate average sum rate. The multiuser connectivity model facilitates a more realistic study of the APC and EE performance under blocking constraints.

2) Multi-user Connectivity Model: Under this model, each BS can connect multiple users depending on the available number of channels. Assume that the system bandwidth $\mathcal{B}$ $\mathrm{Hz}$ is subdivided into $\delta$ channels, each of size $\mathcal{B}_{\delta} \mathrm{Hz}$. Then, each BS can connect up to a maximum of $N=\delta$ users to avoid intra-cell interference.

Theorem 1. The ACR in the multi-user connectivity model is expressed as [22]

$$
\operatorname{ACR}(v, \delta)=\frac{\lambda_{b}}{\lambda_{u}} \times \mathbb{E}\left[C_{b}\right]
$$

where $C_{b} \in\{0, \delta\}$ is the number of served users in the cell and $\mathbb{E}\left[C_{b}\right]$ is expressed as

$$
\mathbb{E}\left[C_{b}\right]= \begin{cases}\mathbb{P}(N \geq \delta), & \delta=1 \\ \delta \mathbb{P}(N \geq \delta)+\sum_{k=1}^{\delta-1} k \mathbb{P}(N=k), & \delta>1\end{cases}
$$

Proof. See Appendix A.

Special Case 1. When $\delta=1, \mathbb{E}\left[C_{b}\right]=p_{a}$ which gives the single connectivity model.

According to (12), the required BS density to achieve the blocking constraint $\chi$ must satisfy the expression $\lambda_{b} \mathbb{E}\left[C_{b}\right] \geq$ $(1-\chi) \lambda_{u}$. To illustrate the importance of this model, we apply the same constraint $\chi=0.1$ and consider the simple case of $\delta=2$. Then,

$$
\mathbb{E}\left[C_{b}\right]_{\{\delta=2\}}=2 p_{a}-\frac{\lambda_{u}}{\lambda_{b}}\left(1+\frac{\lambda_{u}}{\mathcal{K} \lambda_{b}}\right)^{-(\mathcal{K}+1)} .
$$

Using bisection method, the required BS density $\lambda_{b} \geq 1.35 \times$ $10^{-4} \mathrm{~m}^{2}$ such that $\lambda_{b}|\mathcal{A}| \geq 844$ BSs. This is in contrast to the over $4000 \mathrm{BSs}$ required in the single user connectivity model. As $\delta$ increases, the BS-user density ratio continues to reduce and becomes evermore realistic. The ACR performance of the network is affected by the applied sleep mode strategy as discussed later in Section VI.

We will now analyze the average rate performance of the network. Allocating all available channels to the connected users constitutes the upper bound on bandwidth utilization. For each connected user, the density of interfering BSs, denoted as $\bar{p}_{a} \lambda_{b}$, includes all active BSs except its parent BS $b_{o}$ i.e. $\left\{\bar{p}_{a} \lambda_{b}\right\}=\left\{p_{a} \lambda_{b} \backslash b_{o}\right\}$. Consider two scenarios:

1) If $N<\delta$, the BS allocates all channels to the users sequentially. Initially, each user is allocated $\delta_{u}=\lfloor\delta / N\rfloor$ channels and the remaining channels, equal to $\delta_{r}=\delta-$ $\delta_{u} N$, are allocated to any $\delta_{r}$ users chosen randomly from the $N$ users. For example, if $N=4$ and $\delta=10$, two of the users get 3 channels and the other two get 2 channels.

2) If $N \geq \delta$, the BS randomly selects $\delta$ users and allocates a single channel to each one.

Theorem 2. The average rate supported on a typical channel is expressed as

$$
\mathcal{R}_{c h}=\frac{\mathcal{B}}{\delta} \pi \lambda_{b} \Xi\left[e^{-\pi \lambda_{b}\left(1+\bar{p}_{a} \zeta(t, \alpha)\right) z} e^{-\frac{\sigma^{2}}{P_{t} L}\left(2^{t}-1\right) z^{\alpha / 2}}\right]
$$

where $\Xi[f(t, z)]=\int_{t>0} \int_{z>0}[f(t, z)] d z d t$ and $\zeta(t, \alpha)=\left(2^{t}-1\right)^{2 / \alpha} \int_{\left(2^{t}-1\right)^{-2 / \alpha}}^{\infty} \frac{1}{1+u^{\alpha / 2}} \mathrm{~d} u$.

Proof. See Appendix B.

Corollary 1. In an interference-limited network, average channel rate $\overline{\mathcal{R}}_{\text {ch }}$ simplifies to

$$
\overline{\mathcal{R}}_{c h}(v)=\frac{\mathcal{B}}{\delta} \int_{t>0} \frac{1}{1+\bar{p}_{a} \zeta(t, \alpha)} \mathrm{d} t .
$$

Proof. Let $\sigma^{2}=0$ in (14) and simplify the integral. 
Special Case 2. When $\lambda_{u} \gg \lambda_{b}, \bar{p}_{a} \approx 1$ and $\overline{\mathcal{R}}_{c h}$ further simplifies to

$$
\overline{\mathcal{R}}_{c h}=\frac{\mathcal{B}}{\delta} \int_{t>0} \frac{1}{1+\zeta(t, \alpha)} \mathrm{d} t .
$$

Hence, $\overline{\mathcal{R}}_{c h}$ is invariant with the BS density which is consistent with the result in $[27,(16)]$.

Lemma 1. The average number of channels per connected user is expressed as

$$
\omega=\frac{p_{a} \delta}{\mathbb{E}\left[C_{b}\right]} .
$$

Proof. See Appendix C.

Corollary 2. The average rate of a typical user in the network is expressed as

$$
\mathcal{R}_{u}=\frac{p_{a} \mathcal{B}}{\mathbb{E}\left[C_{b}\right]} \pi \lambda_{b} \Xi\left[e^{-\pi \lambda_{b}\left(1+\bar{p}_{a} \zeta(t, \alpha)\right) z} e^{-\frac{\sigma^{2}}{P_{t} L}\left(2^{t}-1\right) z^{\alpha / 2}}\right] .
$$

Proof. $\mathcal{R}_{u}$ is determined from Theorem 2 and Lemma 1 as $\mathcal{R}_{u}=\omega \cdot \mathcal{R}_{c h}$.

When $\sigma^{2}=0$, the average user rate of the interferencelimited network simplifies to

$$
\overline{\mathcal{R}}_{u}=\omega \cdot \overline{\mathcal{R}}_{c h}=\frac{p_{a} \mathcal{B}}{\mathbb{E}\left[C_{b}\right]} \int_{t>0} \frac{1}{1+\bar{p}_{a} \zeta(t, \alpha)} \mathrm{d} t .
$$

Average network sum rate $\mathcal{T}$ is determined from $\mathcal{R}_{u}$ in (18) and the connected users as

$$
\mathcal{T}=\mathrm{ACR} \times \lambda_{u} \times \mathcal{R}_{u}=\lambda_{b} \mathbb{E}\left[C_{b}\right] \mathcal{R}_{u} .
$$

For the interference-limited case, $\overline{\mathcal{T}}$ is determined accordingly using (19).

\section{B. Probability of Coverage}

The coverage probability of a typical user in the network is expressed as [27]

$$
\mathcal{P}_{c}=\pi \lambda_{b} \int_{s>0} e^{-\pi \lambda_{b}\left(1+\bar{p}_{a} \rho(T, \alpha)\right) s} e^{-\frac{T \sigma^{2}}{P_{t} L} s^{\alpha / 2}} \mathrm{~d} s
$$

where $\rho(T, \alpha)=T^{2 / \alpha} \int_{T^{-2 / \alpha}}^{\infty} \frac{1}{1+u^{\alpha / 2}} \mathrm{~d} u$ and $T$ is the SINR coverage threshold. Unlike the analysis in [27], we consider the possibility of idle BSs which thin the aggregate interference.

In the interference-limited network, coverage probability $\overline{\mathcal{P}}_{c}$ simplifies to

$$
\overline{\mathcal{P}}_{c}(v, T, \alpha) \stackrel{(a)}{=} \frac{1}{1+\bar{p}_{a} \rho(T, \alpha)} \stackrel{(b)}{=} \frac{1}{1+\rho(T, \alpha)}
$$

where $(a)$ shows that $\overline{\mathcal{P}}_{c}$ is also heavily influenced by the prevailing BS-user density ratio $v$. In addition, $(b)$ describes the special case of $\lambda_{b} \ll \lambda_{u}$ where $p_{a} \approx 1$ such that $\overline{\mathcal{P}}_{c}$ is independent of the BS density. This special case scenario is analyzed in [27].

Lemma 2. When $\sigma^{2}>0$ and $\alpha>2$ which is true for a mobile environment, both coverage probability and average user rate increase monotonically with the $B S$ density and transmit power.

Proof. See Appendix D.

\section{CONSTRAINED OPTIMIZATION FRAMEWORK}

In the last section, we analyzed the PPP-based homogeneous network in terms of its coverage and average rate performance. We now consider two approaches to managing the network energy performance: (a) In this section, we determine the initial optimal deployment strategy of the network in terms of its deployment factor [15]. In this case, the BS density and transmit power in the network are jointly optimized to determine what optimal combination minimizes the APC; (b) Once the network is in existence, the sleep mode schemes which are discussed in Section V are then applied to adapt network power consumption to the prevailing user density or traffic intensity. The idea is that even if the network is initially deployed with optimal parameters, it still exists in an environment of highly variable spatiotemporal distribution of users or traffic and hence, there are more energy saving opportunities.

In this paper, the deployment factor, expressed as $\mathcal{H}=$ $\lambda_{b}^{\alpha / 2} P_{t}$, is simply a mathematical expression that combines the required BS density and associated transmit power that achieve a given performance constraint. Therefore, the deployment factor $\mathcal{H}$ can be optimized to determine the specific combination of BS density and transmit power that minimizes the APC, which is this paper's main objective. This joint optimization of BS density and transmit power provides a flexible framework where for instance BS density can be reduced if the transmit power is increased by an appropriate determinable amount.

Both coverage probability and average user rate are considered as the performance constraints during network optimization. Both constraints are necessary because satisfying one does not automatically satisfy the other. For instance, (i) if SINR is high (good coverage), average user rate may remain low due to insufficient bandwidth; and (ii) if SINR is low (poor coverage), average user rate may still be high due to high bandwidth availability.

As more BSs are deployed in the network, aggregate interference increases significantly and becomes dominant over noise, making the interference-to-noise ratio (INR) high. In this case, the network is essentially interference-limited, making the effect of noise anywhere in the network negligible [27]. Therefore, the interference-limited network defines the upper bound performance of coverage probability and average rate of a general network. In this paper, we express the coverage probability and average rate constraints as functions of their respective upper bounds as follows:

$$
\mathcal{P}_{c}=\epsilon \overline{\mathcal{P}}_{c} \quad \text { and } \quad \mathcal{R}_{u}=\kappa \overline{\mathcal{R}}_{u}
$$

respectively, where $\epsilon \in(0,1]$ and $\kappa \in(0,1]$ are the ratios of the coverage probability and average user rate to their respective upper bound values. The parameters $\epsilon$ and $\kappa$ are set by the operator based on the desired coverage and average rate performance. It should be noted however that as $\epsilon$ and $\kappa$ increase, so does the BS density and/or transmit power required to push $\mathcal{P}_{c}$ and $\mathcal{R}_{u}$ closer to their upper bound levels $\overline{\mathcal{P}}_{c}$ and $\overline{\mathcal{R}}_{u}$ respectively. 


\section{A. Coverage Probability Constraint}

Corollary 3. When $\sigma^{2}>0$, the coverage probability in (21) can be approximated as

$$
\mathcal{P}_{c} \approx \overline{\mathcal{P}}_{c}\left(1-\frac{T \sigma^{2} \psi(\alpha) \overline{\mathcal{P}}_{c}^{\alpha / 2}}{\lambda_{b}^{\alpha / 2} P_{t}}\right),
$$

where $\psi(\alpha)=\frac{\Gamma\left(\frac{\alpha}{2}+1\right)}{\pi^{\alpha / 2} L}$.

Proof. See Appendix E. Fig. 1(a) shows a tight approximation in a dense network.

Using (23) and (24) and Lemma 2, the coverage probability constraint can be rewritten as

$$
\frac{\mathcal{H}_{c}^{\star}}{\overline{\mathcal{P}}_{c}^{\alpha / 2}}-\frac{T \sigma^{2} \psi(\alpha)}{(1-\epsilon)}=0
$$

where $\mathcal{H}_{c}^{\star}=\lambda_{b}^{\alpha / 2} P_{t}$ is the deployment factor that satisfies the coverage probability constraint.

In general, it is not possible to find a closed form expression for $\mathcal{H}_{c}^{\star}$ in (25) because $\overline{\mathcal{P}}_{c}$ is also a function of $\lambda_{b}$. To find the optimal combination of $\lambda_{b}$ and $P_{t}$ that minimizes APC, we use different values of $P_{t}$ and then determine the corresponding values of $\lambda_{b}$ using bisection method. Working backwards, we then determine the APC for each combination of $P_{t}$ and $\lambda_{b}$. Over a wide range of $P_{t}$ values, we then determine the combination of $P_{t}$ and $\lambda_{b}$ that minimizes APC and denote it as $\left(\lambda_{b}^{\star}, P_{t}^{\star}\right)$. Although this approach is not perfectly accurate, it allows us to obtain insights into a problem that would otherwise be impossible to solve. This approach, although rather involved, is reasonable since network planning and optimization tasks are generally performed offline. It is however possible to express the optimal deployment factor in closed form in the following special scenario.

Special Case 3. When $\lambda_{b} \ll \lambda_{u}, p_{a} \approx 1$ and $\overline{\mathcal{P}}_{c}$ is independent of $\lambda_{b}$. Hence (25) simplifies to

$$
\mathcal{H}_{c}^{\star}=\frac{T \sigma^{2} \psi(\alpha)}{(1-\epsilon)} \overline{\mathcal{P}}_{c}^{\alpha / 2} .
$$

To minimize APC, $\mathcal{H}_{c}$ is optimized as a bivariate problem using the following framework:

$$
\begin{cases}\underset{\lambda_{b}, P_{t}}{\operatorname{minimize}} & \lambda_{b}\left(N_{t x} P_{0}+\Delta P_{t}\right) \\ \text { subject to } & \lambda_{b}^{\alpha / 2} P_{t}=\mathcal{H}_{c}^{\star}, P_{t} \leq \overline{P_{t} .}\end{cases}
$$

This problem can easily be converted into a single variable problem and its solutions are

$$
P_{t}^{\star}=\min \left\{\frac{2 N_{t x} P_{0}}{\Delta(\alpha-2)}, \overline{P_{t}}\right\} ; \quad \lambda_{b, c}^{\star}=\left(\frac{\mathcal{H}_{c}^{\star}}{P_{t}^{\star}}\right)^{2 / \alpha} .
$$

\section{B. Average User Rate Constraint}

Corollary 4. When $\sigma>0$, the average user rate in (18) can be approximated as

$$
\mathcal{R}_{u} \approx \overline{\mathcal{R}}_{u}-\frac{\mathcal{B} p_{a} \sigma^{2} \psi(\alpha) \psi_{r}(t, \alpha)}{\mathbb{E}\left[C_{b}\right] \lambda_{b}^{\alpha / 2} P_{t}}
$$
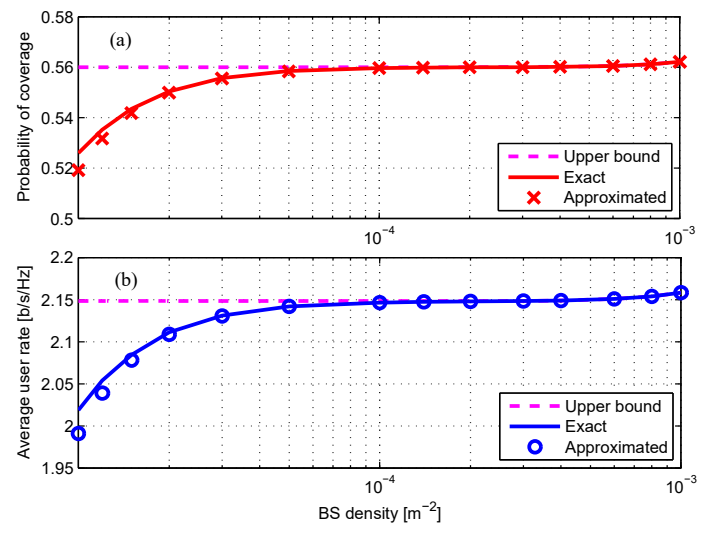

Fig. 1. Exact and approximated (a) coverage probability and (b) average user rate $\left(\lambda_{u}=10^{-2} \mathrm{~m}^{-2}, \alpha=4\right.$, and $\left.T=0 \mathrm{~dB}\right)$.

where $\psi_{r}(t, \alpha)=\int_{t>0} \frac{\left(2^{t}-1\right)}{\left[1+\bar{p}_{a} \zeta(t, \alpha)\right]^{\frac{\alpha}{2}+1}} \mathrm{~d} t$.

Proof. See Appendix F. Fig. 1(b) shows a tight approximation in a dense network.

Combining (23), (29) and Lemma 2, the average user rate constraint can be rewritten as

$$
\frac{\mathcal{H}_{r}^{\star}}{\psi_{r}(t, \alpha)}-\frac{\sigma^{2} \psi(\alpha)}{(1-\kappa) \bar{\Re}}=0,
$$

where $\mathcal{H}_{r}^{\star}=\lambda_{b}^{\alpha / 2} P_{t}$ is the optimal deployment factor that satisfies the rate constraint, and $\bar{\Re}=\int_{t>0} \frac{1}{1+\bar{p}_{a} \zeta(t, \alpha)} \mathrm{d} t$ is the average spectral efficiency when $\sigma^{2}=0$ and $\delta=1$ [27]. Similar to the coverage constraint, it is not possible to find a closed form expression for $\mathcal{H}_{r}^{\star}$ in (30) because both $\psi_{r}(t, \alpha)$ and $\bar{\Re}$ also depend on $\lambda_{b}$. For further analysis, various values of $P_{t}$ are used to determine corresponding values of $\lambda_{b}$. The optimal configuration, denoted as $\left\{\lambda_{b}^{\star}, P_{t}^{\star}\right\}$, is then the combination of $\lambda_{b}$ and $P_{t}$ that minimizes APC.

Special Case 4. When $\lambda_{b} \ll \lambda_{u}, \psi_{r}(t, \alpha)$ and $\bar{\Re}$ are independent of $\lambda_{b}$. Hence (30) simplifies to

$$
\mathcal{H}_{r}^{\star}=\frac{\sigma^{2} \psi(\alpha) \psi_{r}(t, \alpha)}{(1-\kappa) \bar{\Re}} .
$$

Therefore $\mathcal{H}_{r}$ is in closed form and the optimal BS density and transmit power are determined by solving a similar bivariate problem as in (27)-(28). The solutions are

$$
P_{t}^{\star}=\min \left\{\frac{2 N_{t x} P_{0}}{\Delta(\alpha-2)}, \overline{P_{t}}\right\} ; \quad \lambda_{b, r}^{\star}=\left(\frac{\mathcal{H}_{r}^{\star}}{P_{t}^{\star}}\right)^{2 / \alpha} .
$$

According to (28) and (32), $P_{t}^{\star}$ is independent of both $\epsilon$ and $\kappa$ and only depends on the BS power consumption parameters. Hence, $P_{t}^{\star}$ can be predetermined if the BS type is known.

\section{Overall Solution}

Coverage and average rate constraints are complementary to each other because optimization based on one measure also improves the other measure. Since $P_{t}^{\star}$ is similar for both constraints, the overall optimal BS density is the one that satisfies both constraints simultaneously i.e.

$$
\lambda_{b}^{\star}=\max \left\{\lambda_{b, c}^{\star}, \lambda_{b, r}^{\star}\right\} .
$$




\section{Sleep Mode Strategies}

Cellular subscribers are distributed unevenly within the network in both space and time dimensions. For example, urban areas have a high concentration of users during the day but they move to suburban residential areas at night. Similarly, more traffic is originated during the mid-morning and evening hours than late at night [12]. This causes significant traffic load variation over each 24-hour day. According to [11] for example, only $10-30 \%$ of subscribers are usually active during the busy periods. Furthermore, a study of mature markets by GreenTouch [37] shows that a typical traffic profile varies between $20 \%$ and $140 \%$ of the average traffic load. This traffic load profile can be arranged into a load-level step function with five steps as shown in Fig. 2 [37]. This inevitable and fairly predictable traffic variation provides a great opportunity for saving energy by adapting the active BS density to the prevailing traffic load level.

In this paper, we assume that the BS on/off switching is based on a long term traffic profile as in [38]. Over each traffic load-level segment, an appropriate active BS density is determined to serve the prevailing active subscribers; all other BSs are switched off (i.e. deep sleep mode) for the entire duration of this segment. The active BSs provide coverage over the entire network area, usually at the cost of slightly increased transmit power to serve some distant users. However, this approach is reasonable especially in dense urban scenarios where the overall BS density is high and thus a typical user will not be too far away from an active BS. Depending on the varying spatial distribution of users within each traffic load segment, it is still possible that some BSs can be idle some of the time. In such a case, the network puts such BSs in light sleep mode, waking them up quickly when required to respond to short-term traffic variations.

The criteria followed in choosing BSs for sleep mode highly impacts network performance. In this section, we propose a new sleep mode scheme called centralized strategic scheme and investigate its energy consumption performance. To ease implementation of the strategic algorithm, we also propose a distributed strategic sleep mode scheme and compare its performance to that of its optimal centralized counterpart. Two other sleep mode schemes from literature are used for comparison purposes in this paper: (i) conventional sleep mode which has already been discussed in section IV; and (ii) random sleep mode which was introduced in [18]. All schemes are analyzed in terms of their coverage, average rate and energy performance.

\section{A. Conventional Sleep Mode}

This sleep mode approach only responds to real-time distribution of traffic and puts all idle BSs of density $\left(1-p_{a}\right) \lambda_{b}$ to light sleep mode to save energy. No BSs are put in deep sleep mode for a predefined period in response to the traffic load profile of Fig. 2. Since all connected users maintain their original parent BSs, they also maintain their original received signal strength. However, aggregate interference is thinned out by the BSs in light sleep mode which enhances average SINR in the network. Under this scheme, the probability of active

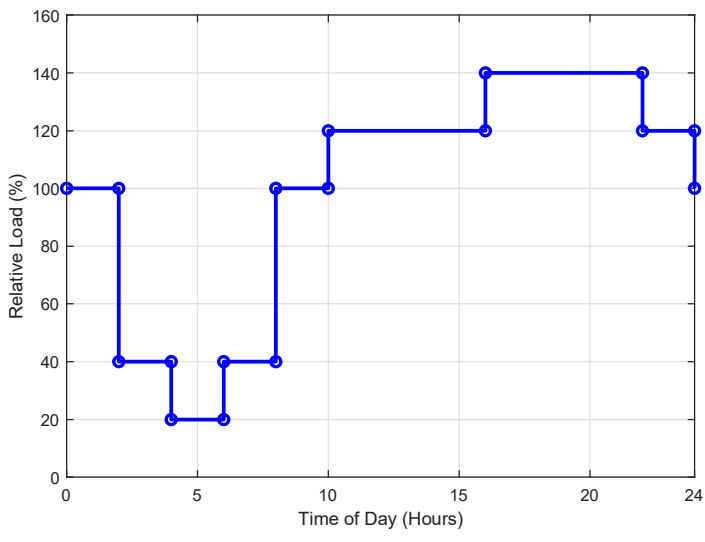

Fig. 2. Traffic profile for a dense urban area [37].

BS density is shown in (7) and the corresponding APC in (3). All the preceding network analysis in Sections III and IV is based on this scheme.

\section{B. Random Sleep Mode}

Although conventional sleep mode puts all idle BS to light sleep mode, it may become necessary to switch off more BSs to reduce energy consumption and enhance EE. In random sleep mode, once the density of BSs required to satisfy a given traffic load level has been determined, the available BS density is independently thinned and the selected BSs are put in deep sleep mode. Therefore, some users are forced to connect to more distant BSs if their nearest (original parent) BSs are switched off. The multi-user connectivity model facilitates the thinned BS density to connect more users and maintain an acceptable blocking rate.

If $p_{r}$ is the target proportion of available $\mathrm{BSs}$ for cell association, then the density of BSs put in deep sleep mode is $\left(1-p_{r}\right) \lambda_{b}$. Since the available BS density is $p_{r} \lambda_{b}$, random and conventional sleep modes are equivalent only when $p_{r}=1$. After cell association, there is a finite probability that some BSs may still remain idle due to short-term traffic variations. Such idle BSs are only put in light sleep mode as they may will be required more regularly to respond to short-term traffic variations. Therefore, the active BS probability with random sleep mode is expressed as

$$
p_{a_{r}}=1-\mathbb{P}\left(N_{r}=0\right)=1-\left(1+\frac{\lambda_{u} / p_{r} \lambda_{b}}{\mathcal{K}}\right)^{-\mathcal{K}},
$$

where $N_{r}$ is the number of users in a cell. Active BS density is then $p_{a_{r}} p_{r} \lambda_{b}$ and the density of idle BSs is $\left(1-p_{a_{r}}\right) p_{r} \lambda_{b}$. Hence, the APC of the network is determined as

$$
\mathrm{APC}=p_{r} \lambda_{b}\left[p_{a_{r}}\left(P_{\text {Act }}-P_{\text {Lsleep }}\right)+P_{\text {Lsleep }}\right] .
$$

The analysis of random sleep mode is essentially similar to that of conventional sleep mode, the only difference being their available BS densities which are $p_{r} \lambda_{b}$ and $\lambda_{b}$ respectively. For example, comparing (7) and (34) shows the similarity in their active BS probability. Due to space constraints, the mathematical analysis of random sleep mode is not shown. 
Unlike random sleep mode, practical schemes are likely to consider the dynamic spatiotemporal distribution of users in the network while selecting BSs for sleep mode. We now propose a centralized strategic sleep algorithm which exploits this spatiotemporal variation of traffic to enhance energy performance. A distributed implementation of the strategic algorithm is also discussed.

\section{Centralized Strategic Sleep Mode}

In random sleep mode, the BSs put in deep sleep mode are selected in a random manner and hence some BSs with many users are also affected which results in a poor performance. In centralized strategic sleep mode, a BS remains active depending on its perceived degree of importance to the network. In practice, the degree of importance of a typical BS can be computed and updated over time by the network; for example, it could be based on the average number of connections that the BS maintains during the busy period. The scheme then prioritizes BSs with the least degree of importance to the network for sleep mode [22]. If the network is initially thinned to $p_{s} \lambda_{b}\left(p_{s}\right.$ is the fraction of all BSs that remain available for cell association), then the density of BSs in deep sleep mode is $\left(1-p_{s}\right) \lambda_{b}$. The value of $p_{s}$ is set using any desired criteria; for example it can be based on a requirement to serve a given traffic load level as in Fig. 2. In addition, it can be set to keep BSs with at least a given average number of connections; for example, if BSs with $N<n$ connections are to be put to sleep, then $p_{s}$ is obtained using (6) as

$$
p_{s}=1-\mathbb{P}(N<n)=\sum_{m=0}^{n-1} \frac{\lambda_{u}^{m}\left(\mathcal{K} \lambda_{b}\right)^{\mathcal{K}} \Gamma(m+\mathcal{K})}{\Gamma(\mathcal{K}) m !\left(\lambda_{u}+\mathcal{K} \lambda_{b}\right)^{m+\mathcal{K}}} .
$$

Due to the mobility of users that causes short-term spatiotemporal traffic variability, the number of connections per active BS is not a constant over the entire period of each load-level segment. To emulate this effect, we assume that a density $f_{u} \lambda_{u}$ (where $0 \leq f_{u} \leq 1$ ) of users have their locations changed in each simulation trial, while the rest are assumed to be stationary. This means that the number of users per BS is changing in each iteration. Furthermore, some BSs may momentarily become idle depending on the values of $p_{s}, f_{u}$ and $\lambda_{u}$. Those BSs are put in light sleep mode within that iteration. Over time however, the strategic algorithm ensures that the number of connected users is maximized for a given active BS density compared to random sleep mode. Assuming that the probability of a BS being idle after a density $f_{u} \lambda_{u}$ of users have changed locations is $\tilde{p}_{a}$, then the instantaneous active BS density is $\tilde{p}_{a} p_{s} \lambda_{b}$ while the BS density in light sleep mode is $\left(1-\tilde{p}_{a}\right) p_{s} \lambda_{b}$. The APC of the network is then expressed as

$$
\mathrm{APC}=p_{s} \lambda_{b}\left[\tilde{p}_{a} P_{\text {Act }}+\left(1-\tilde{p}_{a}\right) P_{\text {Lsleep }}\right] .
$$

\section{Distributed Strategic Sleep Mode}

Although the centralized strategic scheme is optimal, it is difficult to implement in very large networks. In the distributed strategic scheme, the strategic algorithm is implemented in smaller clusters all over the network. The network is subdivided into a grid of $N_{c}$ equal-sized squares where each square represents a cluster area. Cluster boundaries only determine which cluster each BS belongs to for sleep mode optimization and do not affect cell association since users still connect to their nearest parent BSs. Therefore, BSs within each square form a cluster and together with their associated users define the cluster's BS-user density ratio. Although this makes it easier and more manageable to implement, it trades off some of the performance of the centralized strategic scheme. If $N_{c}=1$, the centralized and distributed strategic sleep mode schemes are identical.

If the $i$-th cluster has a BS density $\lambda_{b_{i}}$, the respective BS densities $p_{s} \lambda_{b_{i}}$ and $\left(1-p_{s}\right) \lambda_{b_{i}}$ remain awake or go to deep sleep mode respectively based on their degree of importance to the network. For consistency, the change in locations of a density $f_{u} \lambda_{u}$ of the users is done over the whole network and not within each individual clusters. Similarly, there is a chance that some of the remaining BSs can become idle; these are put in light sleep mode. Hence, the BS densities in active and light sleep mode are $\tilde{p}_{a_{i}} p_{s} \lambda_{b_{i}}$ and $\left(1-\tilde{p}_{a_{i}}\right) p_{s} \lambda_{b_{i}}$ respectively, where $\tilde{p}_{a_{i}}$ is the probability of a BS in the $i$-th cluster not being idle. The network APC with distributed sleep mode is then expressed as

$$
\mathrm{APC}=\sum_{i=1}^{N_{c}} p_{s} \lambda_{b_{i}}\left[\tilde{p}_{a_{i}} P_{\text {Act }}+\left(1-\tilde{p}_{a_{i}}\right) P_{\text {Lsleep }}\right] .
$$

It is important to investigate how the distributed strategic scheme affects the performance of the strategic algorithm compared to the centralized strategic scheme. In the centralized scheme, the optimal set of available BS, denoted as $\widetilde{\mathbf{B}}_{c}$, contains the best BSs in terms of degree of importance. For the distributed scheme, denote the set of available BSs in the $i$-th cluster as $\widetilde{\mathbf{B}}_{d_{i}}=\left\{p_{s} \lambda_{b_{i}}\right\}$. If the overall set of available BSs is denoted as $\widetilde{\mathbf{B}}_{d}$, then $\widetilde{\mathbf{B}}_{d}=\cup_{i=1}^{N_{c}} \widetilde{\mathbf{B}}_{d_{i}}$. Due to the spatiotemporal distribution of BSs and users, a given cluster may contain many important BSs while another cluster has few such BSs. Therefore, some clusters may be forced to leave out important BSs while others contribute unimportant ones. This unwanted outcome gets worse as the density of clusters increases. In other words, we can conclude that $\widetilde{\mathbf{B}}_{c} \neq \widetilde{\mathbf{B}}_{d}$. The suboptimal composition of set $\widetilde{\mathbf{B}}_{d}$ explains the suboptimal performance of the distributed strategic scheme compared to its centralized counterpart. However, results in Section VI show only a small performance degradation.

\section{NumericAl RESUlts}

This section discusses the performance of the PPP-based homogeneous network with the default parameters shown in Table II, unless otherwise stated.

As Fig. 3 shows, average channel rate $\mathcal{R}_{c h}$ depends on the prevailing BS-user density ratio $v$. Coverage probability also follows the same trend. There are two $v$-ranges of interest:

1) At low $v$ (where $\lambda_{u} \gg \lambda_{b}$ ), $\overline{\mathcal{R}}_{c h}$ in the interferencelimited network ("no-noise" scenario) is invariant with the BS density since all BSs remain active. This is consistent with the analysis in [27]. In addition, $\overline{\mathcal{R}}_{c h}$ 
TABLE II

SIMULATION PARAMETERS

\begin{tabular}{|l|l|}
\hline \hline Parameters & Values \\
\hline Network size $\mathcal{A}$ & $5 \mathrm{~km} \times 5 \mathrm{~km}$ \\
\hline System bandwidth $\mathcal{B}$ & $20 \mathrm{MHz}$ based on a LTE network \\
\hline BS and user densities & $\lambda_{b}=1.6 \times 10^{-5} \mathrm{~m}^{-2}, \lambda_{u}=2 \lambda_{b}$ \\
\hline BS transmit power & $P_{t}=21 \mathrm{dBm}$ \\
\hline Pathloss parameters & $L=-33 \mathrm{~dB}, \alpha=4$ \\
\hline BS power parameters & $N_{t x}=2, P_{0}=6.8 \mathrm{~W}, \Delta=4, P_{s l}=4.3 \mathrm{~W}$ \\
\hline Noise parameters & $F=10, T_{a}=300 \mathrm{~K}$ \\
\hline Percentage of mobile users & $f_{u}=50 \%$ \\
\hline
\end{tabular}

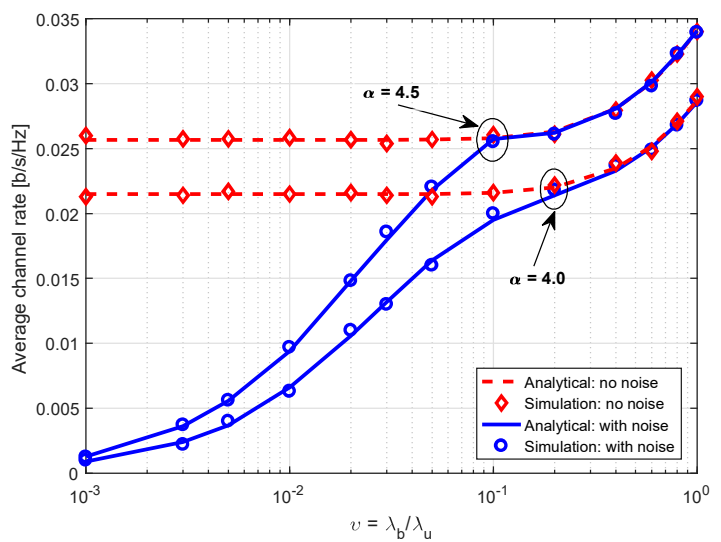

Fig. 3. Average channel rate against $v$ in a network using conventional sleep mode (for $\lambda_{u}=10^{-3} \mathrm{~m}^{-2}$ ).

increases with $\alpha$ because a higher $\alpha$ attenuates interference signals (that originate further away) more than the received signal and this increases the average SINR in the network. In the presence of noise however, the network is essentially noise-limited since interference is very low due to a low BS density. Hence, $\mathcal{R}_{c h}$ is considerably lower than its upper bound $\overline{\mathcal{R}}_{c h}$. In contrast, $\mathcal{R}_{c h}$ now reduces as $\alpha$ increases because the received power degrades more at a higher $\alpha$.

2) As $v$ increases (or $\lambda_{b}$ increases), the gradual increase in aggregate interference eventually makes the network interference-limited and the "with-noise" and "no-noise" characteristics eventually merge. When $v$ increases further, idle BSs begin to appear and their thinning effect on interference enhances the rate in both scenarios.

Fig. 4 shows that $\lambda_{b, c}^{\star}$ and $\lambda_{b, r}^{\star}$ increase with their respective constraints $\epsilon$ and $\kappa$ because the network requires more BSs to get closer to its interference-limited state. In addition, $\lambda_{b, c}^{\star}$ increases with $T$ because more BSs are required to enhance coverage probability to a higher threshold $T$. When $T=0 \mathrm{~dB}$, $\lambda_{b, c}^{\star} \approx \lambda_{b, r}^{\star}$. However, when $T>0 \mathrm{~dB}, \lambda_{b, c}^{\star}>\lambda_{b, r}^{\star}$; conversely when $T<0 \mathrm{~dB}, \lambda_{b, c}^{\star}<\lambda_{b, r}^{\star}$. Hence, it is necessary to consider both coverage and rate constraints.

Furthermore, $\lambda_{b, c}^{\star}$ and $\lambda_{b, r}^{\star}$ also vary in different network environments as shown in Fig. 5. Generally, $\lambda_{b, c}^{\star}$ and $\lambda_{b, r}^{\star}$ increase with $\alpha$ since wireless signals degrade more rapidly in high $\alpha$ environments. To investigate the effect of $\alpha$, assume that $\epsilon=\kappa$ and consider the case of $T=0 \mathrm{~dB}$ where both $\lambda_{b, c}^{\star}$ and $\lambda_{b, r}^{\star}$ are in the same range. When $\alpha<4, \lambda_{b, c}^{\star}>\lambda_{b, r}^{\star}$ but

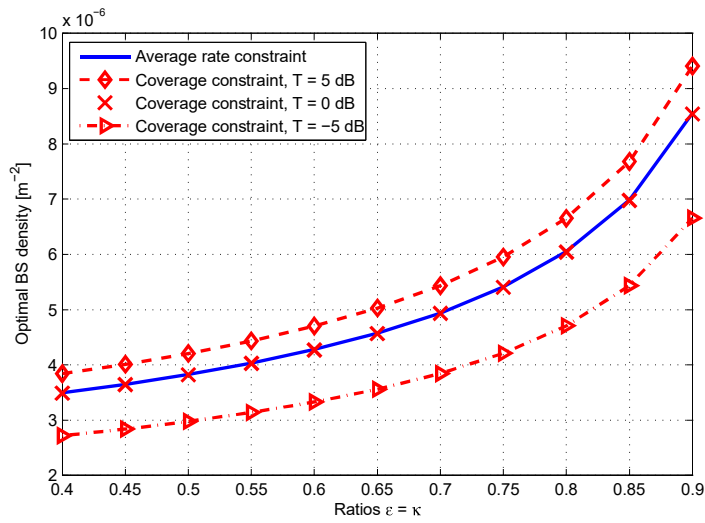

Fig. 4. Optimal BS density against coverage and average rate constraints for $\lambda_{u}=10^{-3} \mathrm{~m}^{-2}$

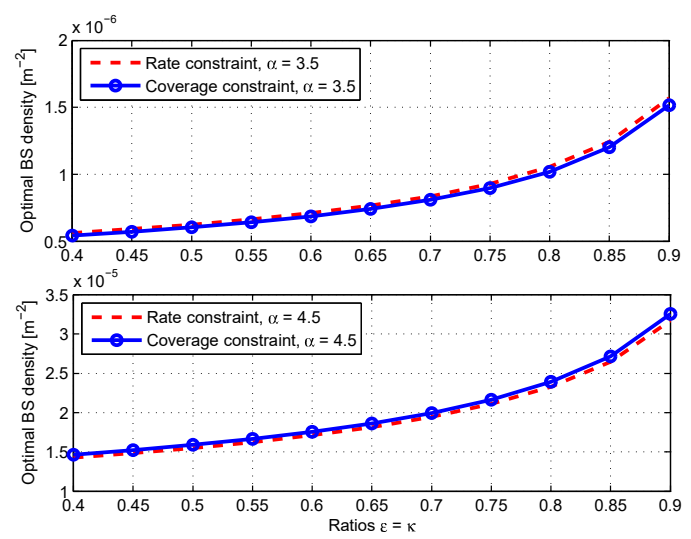

Fig. 5. Effect of path loss exponent on the optimal BS density $\left(\lambda_{u}=\right.$ $\left.10^{-3} \mathrm{~m}^{-2}, T=0 \mathrm{~dB}\right)$.

when $\alpha>4, \lambda_{b, c}^{\star}<\lambda_{b, r}^{\star}$. This also emphasizes the necessity of using both coverage and rate constraints.

The choice of sleep mode strategy has an important bearing on the connectivity performance of the network as shown in Fig. 6. Our multi-user connectivity approach has a better ACR performance than single-user connectivity because it allows multiple users to connect to a BS. The more the number of channels, the higher the ACR value until it reaches its upper bound where every user is connected. In general, conventional sleep mode has the best ACR performance because it maintains the largest BS density for user association. When the BS density is low, the strategic and random sleep mode schemes have comparable ACR performance because most BSs have many more users than they can connect, irrespective of the on/off switching criteria. However, as the BS density increases, the on/off switching approach becomes important and the strategic schemes begin to perform better and their ACR performance eventually approaches that of the conventional scheme. In this high BS density region, the random on/off switching criterion of random sleep mode retains some BSs that contain no users at all and therefore do not contribute to the ACR performance. However, the strategic schemes prioritise BSs with a high likelihood of having users and are therefore able to improve their ACR performance. 


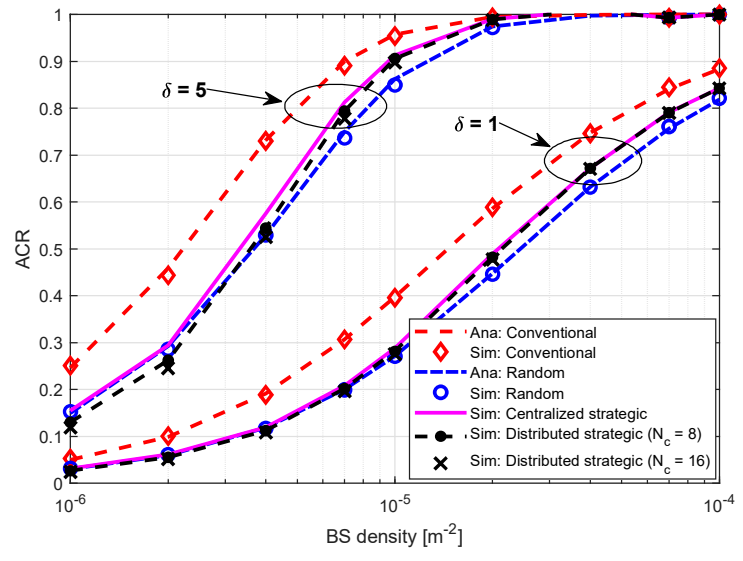

Fig. 6. ACR performance of the different sleep mode strategies, where $p_{s}=$ $0.6, p_{r}=0.6, \lambda_{u}=10^{-5} \mathrm{~m}^{-2}$, and $\delta=\{1,5\}$.

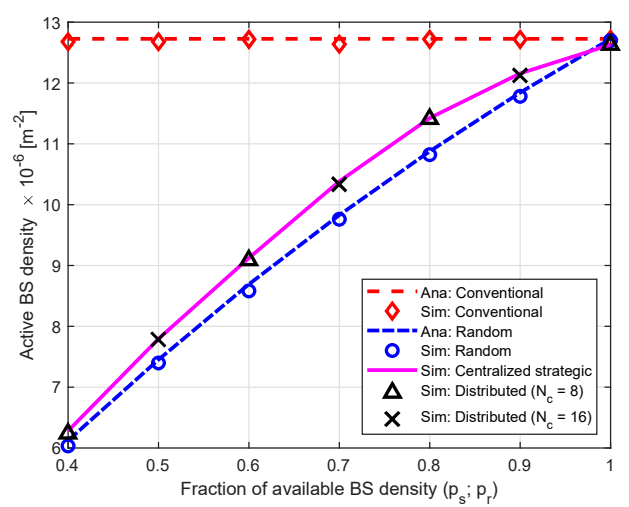

Fig. 7. Active BS density using the different sleep mode schemes.

The coverage probability, average rate and energy consumption of the network are influenced by the sleep mode scheme implemented. Fig. 7 shows the active BS density for each scheme. With both $p_{s}$ and $p_{r}$ equal to 1 , all schemes are identical to the conventional scheme and active BS density is maximized as $p_{a} \lambda_{b}$. Otherwise, centralized strategic sleep mode enhances the density of active BSs which approaches its upper bound (BS density of the conventional scheme) much faster than in the case of random sleep mode. The active BS densities of the distributed and centralized strategic schemes are essentially equal.

Fig. 8 shows that sleep mode generally improves coverage probability. The "no sleep" scenario is a special case which assumes that $\lambda_{u} \gg \lambda_{b}$ (or $p_{a} \approx 1$ ) and is discussed in [27]. In this case, all BSs are active which maximizes aggregate interference in the network thus giving a lower bound on coverage probability. Although switching off BSs increases the average BS-user separation distances, it can lead to improved average SINR if sleep mode is implemented in such a way as to reduce average interference more than it reduces average received signal. In the conventional scheme for example, all users remain connected to their parent BSs which maintains their received signal power but idle BSs are put in light sleep mode which thins interference and consequently enhances SINR. In the centralized strategic scheme, the most

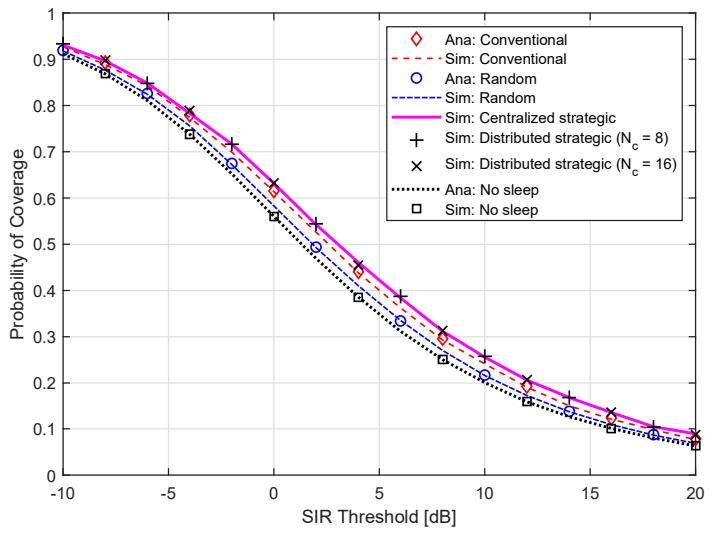

Fig. 8. Coverage probability of the interference-limited homogeneous network with sleep mode $\left(\left\{p_{r}, p_{s}\right\}=0.6<p_{a}\right)$.

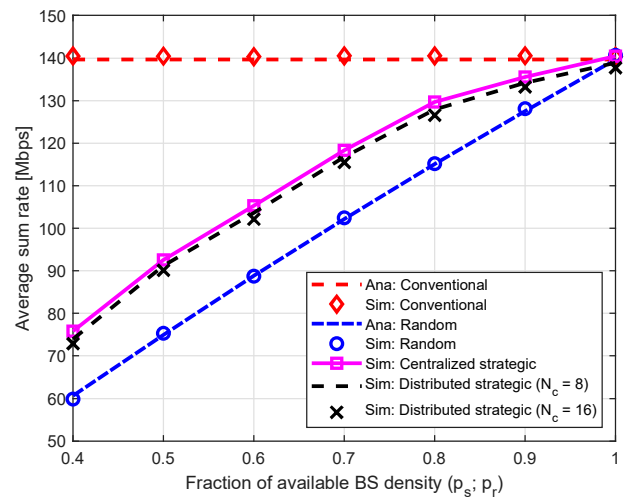

Fig. 9. Average sum rate of a network with sleep mode mechanisms.

important BSs are maintained, meaning that a significant portion of users are not gravely affected by sleep mode which enhances average SINR and improves coverage probability. In distributed strategic scheme, performance is essentially similar to its centralized counterpart because of their comparable BS densities. However, random sleep mode gives only slight improvement of average SINR over the "no-sleep" scheme due to its random criteria of choosing BSs for sleep mode.

Sleep mode schemes also impact the realizable average sum rate of the network as shown in Fig. 9. Conventional sleep mode has a constant average sum rate because it maintains the same average active BS density of $p_{a} \lambda_{b}$. It also defines the upper bound of the average sum rate because of its superior active BS density which maximizes both the number of connected users and the bandwidth per connected user. Random sleep mode has the worst average rate performance because of its random selection of sleep mode BSs. In contrast, centralized strategic sleep mode optimizes the selection process of sleep mode BSs to enhance the average sum rate which approaches its upper bound more rapidly than with random sleep mode. In addition, the centralized strategic scheme gives a better average sum rate than its distributed counterpart due to the optimal selection BSs in set $\left\{\widetilde{B}_{c}\right\}$ based on their degree of importance to the network. To compare the strategic schemes with random scheme, we can for instance consider their sum 


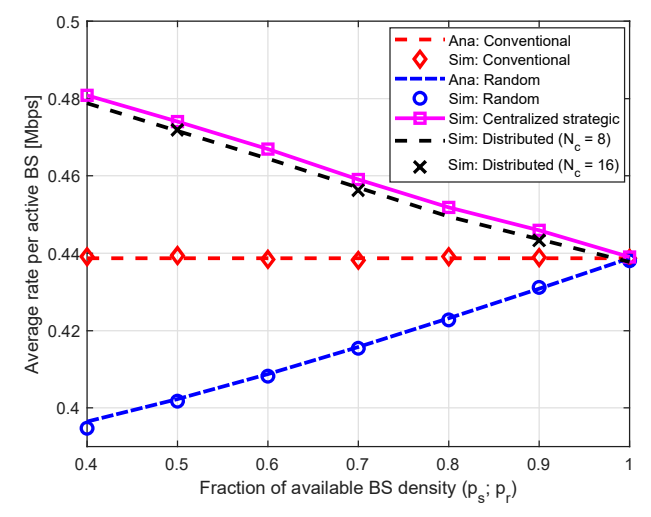

Fig. 10. Average rate per active BS for different sleep mode schemes.

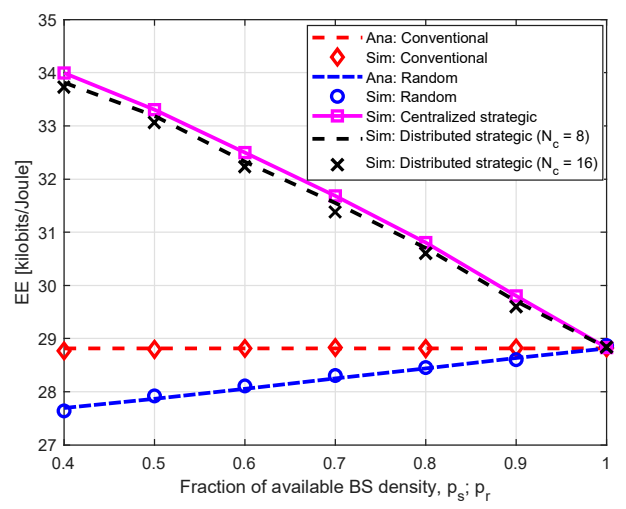

Fig. 11. Network EE performance of different sleep mode schemes.

rate performance at $100 \mathrm{Mbps}$, where random sleep mode requires $68 \%$ of $\mathrm{BSs}$ to be available, while the centralized strategic scheme only needs $56 \%$.

The benefit of sleep mode is to enhance network EE but this is usually achieved at the expense of other performance measures such as average sum rate of the network. However, the best schemes have the ability to maximize the average sum rate from the remaining active BSs. As shown in Fig. 10 , the strategic schemes maximize the average rate of each remaining active BS compared to the conventional and random schemes. Therefore, the strategic schemes give a much improved network EE as shown in Fig. 11. In other words, although conventional sleep mode maximizes the average sum rate as seen in Fig. 9, it does so at the expense of significantly more energy consumption. Random sleep mode has the worst EE performance due to its failure to enhance the average rate from available BSs.

The traffic profile in Fig. 2 is used to evaluate the energy saving potential of the sleep mode schemes. During network planning, operators dimension their networks to meet the peak traffic load requirement, represent by the $140 \%$ load level segment. As the user density reduces in other segments, appropriate BS densities are determined to support the reduced traffic load. Fig. 12 shows the average power saved in the network for the different sleep mode schemes. Conventional sleep mode has the least energy saving because all BSs are

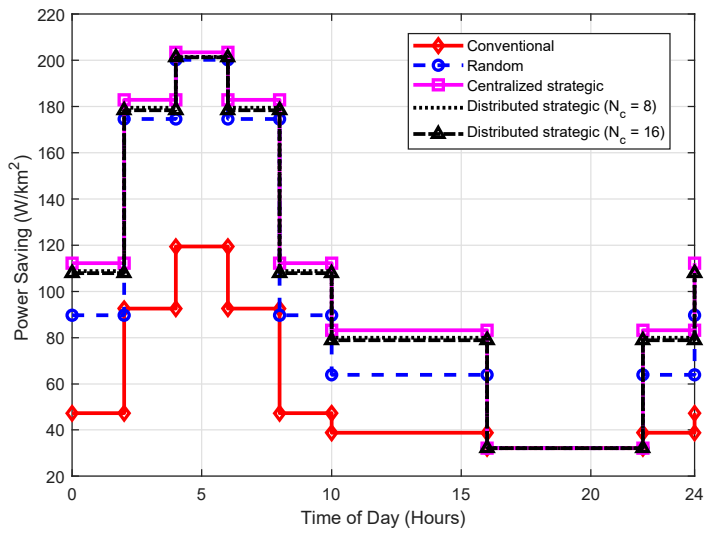

Fig. 12. Power savings of the different sleep mode schemes.

considered for cell association and no BSs are put in deep sleep mode irrespective of the load level. However, BSs of density $\left(1-p_{a}\right) \lambda_{b}$ are idle and are put to light sleep mode to deliver some energy savings. We assume that in the $140 \%$ segment, all BSs are required to serve this high load i.e. $p_{s}=p_{r}=1$ and all schemes resemble conventional sleep mode. As the load reduces, random sleep mode saves more energy than conventional sleep mode because it is able to put some BSs in deep sleep mode. Over the 24-hour period, the random scheme has a 75.5\% energy saving gain over the conventional scheme.

Centralized strategic sleep mode maximizes energy savings because it uses fewer BSs to meet the expected traffic level; it achieves this by maximizing the average rate per active BS as shown in Fig. 10. Over the 24-hour period, this scheme has an energy saving gain of $97 \%$ over the conventional scheme and $13 \%$ over the random scheme. The performance of the distributed strategic scheme is slightly less than its centralized counterpart because of its suboptimal nature. The 24-hour energy saving gains over conventional sleep mode are $92 \%$ and $91 \%$ for the the 8-cluster and 16-cluster topologies respectively. Fewer clusters are less disruptive to the continuity of the network but the performance gap is very negligible. Furthermore, the strategic schemes only have a small advantage over the random scheme in the $20 \%$ and $40 \%$ load level segments. In these low-capacity segments, many BSs are put in deep sleep mode in both scenarios and the few remaining BSs are well loaded irrespective of their selection criteria. However, as the capacity requirements increase and more BSs remain awake, the strategic selection criteria gives a significant benefit over the random selection approach.

\section{CONCLUSIONS}

In this paper, we used the spatial PPP model to analyze the coverage, rate and energy performance of a homogeneous network. We formulated an APC-minimization framework to determine an optimal deployment strategy of the network subject to appropriate coverage and rate constraints. Under special network scenarios, the deployment parameters are expressed in closed form. Analysis showed that optimal transmit power is similar under both coverage and rate constraints. Furthermore, we studied various sleep mode mechanisms to adapt energy 
consumption to variations in traffic load. Results showed that the criteria used in selecting candidate BSs for sleep mode is a major determinant of the overall performance based on coverage, rate and energy consumption. The proposed centralized strategic scheme enhances resource utilization by maximizing the average rate per active BS, which maximizes EE. Over a 24 hour period, it gives a $97 \%$ energy saving gain over conventional sleep mode. Distributing the strategic sleep mode algorithm in clusters all over the network eases implementation and maintains a tight performance approximation compared to the centralized algorithm.

\section{APPENDIX A}

The average number of connected users is expressed as $\operatorname{ACR} \times \lambda_{u}$. This is also equivalent to $\lambda_{b} \times \mathbb{E}\left[C_{b}\right]$, where $\mathbb{E}\left[C_{b}\right]$ is the average number of served users per cell. Hence, $C_{b}=0$ if there are no users and $\delta$ if it has the same or more users than its allocated channels. Since $C_{b}$ takes on non-negative integer values, its expectation is expressed as

$$
\mathbb{E}\left[C_{b}\right]=\sum_{k=1}^{\delta} k \mathbb{P}\left(C_{b}=k\right)
$$

$$
\text { where } \mathbb{P}\left(C_{b}=k\right)= \begin{cases}\mathbb{P}(N=k), & k<\delta \\ \mathbb{P}(N \geq \delta), & k=\delta .\end{cases}
$$

Using different values of $k$ in (39) shows that $\mathbb{E}\left[C_{b}\right]$ is generally expressed as shown in (13).

For Special Case 1, each active BS serves one user. Hence, the number of served users equals the number of active BSs i.e. $\mathrm{ACR} \times \lambda_{u}=\lambda_{b} p_{a}$. Hence, $\mathbb{E}\left[C_{b}\right]=p_{a}$.

\section{APPENDiX B}

The average rate of a channel is expressed as $\mathcal{R}_{c h}=$ $\mathcal{B}_{\delta} \cdot \mathbb{E}\left[\log _{2}(1+\mathrm{SINR})\right]$. Since rate is a non-negative random variable, its expectation is expressed as $\mathbb{E}[X]=\int_{l>0} \mathbb{P}(X>$ $t) \mathrm{d} t$. Following the proof of [27, Theorem 2], $\mathcal{R}_{c h}$ becomes

$$
\begin{aligned}
\mathcal{R}_{c h} & =\frac{\mathcal{B}}{\delta} \int_{t>0} \mathbb{P}\left(\operatorname{SINR}>2^{t}-1\right) \mathrm{d} t \\
& =\frac{\mathcal{B}}{\delta} \Xi\left[e^{-\pi \lambda_{b} r^{\alpha}} e^{\frac{\sigma^{2}}{P_{t} L}\left(2^{t}-1\right)} \mathcal{L}_{I_{i}}\left(r^{\alpha}\left(2^{t}-1\right)\right) \mathrm{d} t 2 \pi \lambda_{b} r \mathrm{~d} r\right]
\end{aligned}
$$

where $\mathcal{L}_{I_{i}}\left(r^{\alpha}\left(2^{t}-1\right)\right)=\exp \left(-\pi\left\{\bar{p}_{a} \lambda_{b} r^{2} \zeta(t, \alpha)\right)\right.$ is the Laplace transform of the thinned interference power. Substituting $z=r^{2}$ gives the result.

\section{APPENDIX C}

Let $\Omega=p_{a} \lambda_{b} \delta$ denote the total number of channels in the network. The average number of connected users $N_{c}=$ $\mathrm{ACR} \times \lambda_{u}=\lambda_{b} \mathbb{E}\left[C_{b}\right]$. Hence $\omega=\Omega / N_{c}$ gives the result.

\section{APPENDIX D}

To prove monotonicity with the BS density, consider two BS densities $\lambda_{b_{1}}$ and $\lambda_{b_{2}}$ where $\lambda_{b_{2}}>\lambda_{b_{1}}$. The probability of coverage corresponding to $\lambda_{b_{2}}$ is

$$
\mathcal{P}_{c}\left(\lambda_{b_{2}}\right)=\pi \lambda_{b_{2}} \int_{0}^{\infty} e^{-\pi \lambda_{b_{2}} A s} \cdot e^{-C s^{\alpha / 2}} \mathrm{~d} s .
$$

where $A=(1+\rho(T, \alpha))$ and $C=\frac{T \sigma^{2}}{P_{t} L}$. Let $s=x \frac{\lambda_{b_{1}}}{\lambda_{b_{2}}}$ and substitute in (40); then

$$
\begin{aligned}
\mathcal{P}_{c}\left(\lambda_{b_{2}}\right) & =\pi \lambda_{b_{1}} \int_{0}^{\infty} e^{-\pi \lambda_{b_{1}} A x} \cdot e^{-C x^{\alpha / 2}\left(\frac{\lambda_{b_{1}}}{\lambda_{b_{2}}}\right)^{\alpha / 2}} \mathrm{~d} x \\
& \stackrel{(a)}{>} \pi \lambda_{b_{1}} \int_{0}^{\infty} e^{-\pi \lambda_{b_{1}} A x} \cdot e^{-C x^{\alpha / 2}} \mathrm{~d} x=\mathcal{P}_{c}\left(\lambda_{b_{1}}\right)
\end{aligned}
$$

where (a) follows since $C>0, \alpha>2$ and $\frac{\lambda_{b_{1}}}{\lambda_{b_{2}}}<1$. Dependence of the average user rate on the BS density is proved in the same way.

To prove monotonicity with the transmit power, consider two transmit power values $P_{t_{1}}$ and $P_{t_{2}}$ where $P_{t_{2}}>P_{t_{1}}$. The coverage probability corresponding to $P_{t_{1}}$ is

$$
\mathcal{P}_{c}\left(P_{t_{1}}\right)=\pi \lambda_{b} \int_{s>0} e^{-\frac{a_{1}}{P_{t_{1}}} s^{\alpha / 2}} e^{-a_{2} s} \mathrm{~d} s,
$$

where $a_{1}=\frac{T \sigma^{2}}{L}$ and $a_{2}=\pi \lambda_{b}\left(1+\bar{p}_{a} \rho(T, \alpha)\right)$. For $P_{t_{2}}$, the coverage probability is

$$
\mathcal{P}_{c}\left(P_{t_{2}}\right)=\pi \lambda_{b} \int_{s>0} e^{-\frac{a_{1}}{P_{t_{2}}} s^{\alpha / 2}} e^{-a_{2} s} \mathrm{~d} s .
$$

Since $a_{1}>0$ and $P_{t_{2}}>P_{t_{1}}$, then $e^{-\frac{a_{1}}{P_{t_{2}}}}>e^{-\frac{a_{1}}{P_{t_{1}}}}$ and hence $\mathcal{P}_{c}\left(P_{t_{2}}\right)>\mathcal{P}_{c}\left(P_{t_{1}}\right)$. The dependence of the average user rate on transmit power is proved in the same way.

\section{APPENDIX E}

Over a realistic SNR range, $T \sigma^{2} \ll P_{t} L$ and the second exponential term in (21) can be approximated as $e^{-\frac{T \sigma^{2}}{P_{t} L} s^{\alpha / 2}} \approx$ $1-\frac{T \sigma^{2}}{P_{l} L} s^{\alpha / 2}$. Substituting this into (21) gives

$$
\begin{aligned}
\mathcal{P}_{c} \approx & \pi \lambda_{b} \int_{s>0} e^{-\pi \lambda_{b}\left(1+\bar{p}_{a} \rho(T, \alpha)\right) s} \mathrm{~d} s- \\
& \pi \lambda_{b} \frac{T \sigma^{2}}{P_{t} L} \int_{s>0} s^{\alpha / 2} e^{-\pi \lambda_{b}\left(1+\bar{p}_{a} \rho(T, \alpha)\right) s} \mathrm{~d} s \\
= & \overline{\mathcal{P}}_{c}-\frac{\pi \lambda_{b} T \sigma^{2} \times \Gamma\left(\frac{\alpha}{2}+1\right)}{P_{t} L\left[\pi \lambda_{b}\left(1+\bar{p}_{a} \rho(T, \alpha)\right)\right]^{\frac{\alpha}{2}+1}} .
\end{aligned}
$$

The second integral is solved using identity [35, (3.381.4)]. Simplifying (43) gives the result.

\section{APPENDIX F}

Since $\sigma^{2} \ll P_{t} L$ in (18), $e^{-\frac{\sigma^{2}}{P_{t} L}\left(2^{t}-1\right) z^{\alpha / 2}} \approx 1-\frac{\sigma^{2}}{P_{t} L}\left(2^{t}-\right.$ 1) $z^{\alpha / 2}$. Hence, $\mathcal{R}_{u}$ can be approximated as

$$
\begin{aligned}
\mathcal{R}_{u} \approx & \frac{p_{a} \mathcal{B}}{\mathbb{E}\left[C_{b}\right]}\left\{\pi \lambda_{b} \Xi\left[e^{-\pi \lambda_{b}\left(1+\bar{p}_{a} \zeta(t, \alpha)\right) z}\right]-\right. \\
& \left.\frac{\pi \lambda_{b} \sigma^{2}}{P_{t} L} \Xi\left[\left(2^{t}-1\right) z^{\alpha / 2} e^{-\pi \lambda_{b}\left(1+\bar{p}_{a} \zeta(t, \alpha)\right) z}\right]\right\} .
\end{aligned}
$$

Evaluating both integrals gives the result. The second integral is solved using [35, (3.381.4)]. 


\section{REFERENCES}

[1] J. Zander and P. Mahonen, "Riding the Data Tsunami in the Cloud: Myths and Challenges in Future Wireless Access," IEEE Communications Magazine, Vol. 51(3), pp. 145-151, March 2013.

[2] Cisco, "Cisco Visual Networking Index: Global Mobile Data Traffic Forecast Update, 2016-2021," White Paper, Feb 2017.

[3] T. Rappaport et. al, "Millimetre Wave Mobile Communications for 5G Cellular: It Will Work!," IEEE Access, Vol.1, pp. 335-349, 2013.

[4] J. G. Andrews et.al, "What will 5G Be", IEEE J. on Sel. Areas in Commun., Vol.32(6), pp. 1065-1082, June 2014

[5] J. G. Andrews, "Seven Ways That HetNets are a Cellular Paradigm Shift," IEEE Commun. Mag., Vol. 51(3), pp. 136-144, March 2013.

[6] S. Sing, H. S. Dhillon, and J. G. Andrews, "Offloading in Heterogeneous Networks: Modeling, Analysis and Design Insights," IEEE Trans. on Wireless Commun., Vol.12(5), pp. 2484-2497, May 2013.

[7] A. Shojaeifard, K. A. Hamdi, E. Alsusa, D. K. C. So, and J. Tang, "A Unified Model for the Design and Analysis of Spatially-Correlated LoadAware HetNets," IEEE Trans. on Communications, Vol.62(11), pp. 41104125, Nov. 2014.

[8] C. Li et al, "Throughput and Energy Efficiency Analysis of Small Cell Networks with Multi-Antenna Base Stations," IEEE Trans. on Wireless Commun., Vol.13(5), pp. 2505-2517, May 2014

[9] Lu Lu, G.Y. Li, A. L. Swindlehurst, A. Ashikhmin, and R. Zhang, "An Overview of Massive MIMO: Benefits and Challenges," IEEE Journ. Sel. Topics in Signal Processing, Vol.8(5), pp. 742-758, Oct. 2014.

[10] H. ElSawy et al., 'Two-Tier HetNets with Cognitive Femtocells: Downlink Performance Modeling and Analysis in a Multi-Channel Environment," IEEE Trans. on Mob. Comp., Vol.13(3), pp. 649-663, Mar. 2014.

[11] G. Auer et al., "How Much Energy is Needed to Run a Wireless Network?," IEEE Wireless Commun. Mag., Vol. 18(5), pp. 40-49, 2011.

[12] M. Marsan et al, "Optimal Energy Savings in Cellular Access Networks," IEEE Intern. Conf. Commun. Wkps pp. 1-5, June 2009

[13] E. Mugume and D.K.C. So, "Spectral and Energy Efficiency Analysis of Dense Small Cell Networks," IEEE Vehicular Technology Conference, pp.1-5, May 2015

[14] D. Cao et al., "Optimal Combination of Base Station Densities for Energy-Efficient Two-Tier Heterogeneous Cellular Networks," IEEE Trans. on Wireless Commun., Vol.12(9), pp. 4350-4362, Sept 2013

[15] J. Peng, P. Hong, and K. Xue, "Energy-Aware Cellular Deployment Strategy Under Coverage Performance Constraints", IEEE Transactions on Wireless Communications, vol. 14, no. 1, pp. 69-80, Jan. 2015.

[16] S. Sarkar, R. K. Ganti and M. Haenggi, "Optimal Base Station Density for Power Efficiency in Cellular Networks", IEEE Intern. Conf. Commun. pp. 4054-4059, June 2014.

[17] X. Wang et al., "Cell Sleeping for Energy Efficiency in Cellular Networks: Is it Viable?," IEEE Wireless Communications and Networking Conf., pp. 2509-2514, April 2012.

[18] Y. Soh, et. al, "Energy Efficient Heterogeneous Cellular Networks," IEEE J. on Sel. Areas in Commun., vol.31(5), pp. 840-850, May 2013.

[19] J. Peng et al, "Stochastic Analysis of Optimal Base Station Energy Saving in Cellular Networks with Sleep Mode," IEEE Commun. Letters, vol.18, no.4, pp. 612-615, April 2014

[20] J. Wu, S. Zhou and Z. Niu, "Traffic-Aware Base Station Sleeping Control and Power Matching for Energy-Delay Tradeoffs in Green Cellular Networks," IEEE Trans. Wireless Communications, vol.12, no.8, pp. 4196-4209, Aug. 2013.

[21] L. Xiang, et.al, "Adaptive Traffic Load-Balancing for Green Cellular Networks", IEEE Intern. Symp. on Personal Indoor and Mobile Radio Commun., pp. 41-45, Sept. 2011

[22] E. Mugume and D.K.C. So, "Sleep Mode Mechanisms in Dense Small Cell Networks," IEEE Intern. Conference on Communications, pp.192197, June 2015

[23] Q. Kuang and W. Utschick, "Energy Management in Heterogeneous Networks With Cell Activation, User Association, and Interference Coordination," IEEE Transactions on Wireless Communications, Vol. 15(6), pp. 3868-3879, 2016

[24] B. Zhuang, D. Guo and M. L. Honig, "Energy-Efficient Cell Activation, User Association, and Spectrum Allocation in Heterogeneous Networks,' IEEE J. on Sel. Areas in Commun., Vol. 34(4), pp. 823-831, 2016.

[25] J. Yang, X. Zhang and W. Wang, "Two-stage base station sleeping scheme for green cellular networks," Journal of Communications and Networks, Vol.18(4), pp. 600-609, Aug. 2016.

[26] P. Chang and G. Miao, "Joint Optimization of Base Station Deep-Sleep and DTX Micro-Sleep," IEEE Globecom Workshops, Washington, DC, 2016, pp. 1-6.
[27] J. G. Andrews, F. Baccelli, and R. K. Ganti, "A Tractable Approach to Coverage and Rate in Cellular Networks," IEEE Transactions on Communications, Vol.59(11), pp. 3122-3134, Nov 2011.

[28] J. Wu, Y. Zhang, M. Zukerman and E. Yung, "Energy-Efficient BaseStations Sleep-Mode Techniques in Green Cellular Networks: A Survey," IEEE Commun. Surveys \& Tutorials, Vol. 17(2), pp. 803-826, April 2015

[29] D. Stoyan, W. Kendall, and J. Mecke, Stochastic Geometry and Its Applications, 2nd Edition, John Wiley and Sons, 1996

[30] M. Haenggi et al., "Stochastic Geometry and Random Graphs for the Analysis and Design of Wireless Networks," IEEE Journal on Selected Areas in Commun., Vol. 27(7), pp. 1029-1046, Sept 2009.

[31] E. Mugume and D. K. C. So, "User Association in Energy-Aware Dense Heterogeneous Cellular Networks," in IEEE Transactions on Wireless Communications, vol. 16, no. 3, pp. 1713-1726, March 2017.

[32] J. G. Andrews, A. K. Gupta, and H. S. Dhillon, "A Primer on Cellular Network Analysis Using Stochastic Geometry," April 2016. Available at: https://arxiv.org/pdf/1604.03183.pdf.

[33] Wei Lu and Marco Di Renzo, "Stochastic Geometry Modeling of Cellular Networks: Analysis, Simulation and Experimental Validation,' June 2015. Available at: http://arxiv.org/pdf/1506.03857v1.pdf.

[34] E. Pineda, and D. Crespo, "Temporal Evolution of the Domain Structure in a Poisson-Voronoi Nucleation and Growth Transformation," Physical Review E, American Physical Society, 2008.

[35] I. S. Gradshteyn and I. M. Ryzhik, Table Of Integrals, Series and Products, 7th Ed., Elsevier Academic Press, 2007.

[36] R. L. Burden and J. D. Faires, Numerical Analysis: Bisection Method, 9th Ed., Brooks/Cole, Cengage Learning, 2011.

[37] O. Blume et al, "Energy Efficiency of LTE Networks Under Traffic Loads of 2020," 10th International Symposium on Wireless Communication Systems, pp. 1-5, 2013.

[38] C. Peng, et al., "Traffic-driven Power Saving in Operational 3G Cellular Networks," Proceedings of the 17th annual international conference on Mobile computing and networking (ACM), pp. 121-132, Sept 2011.

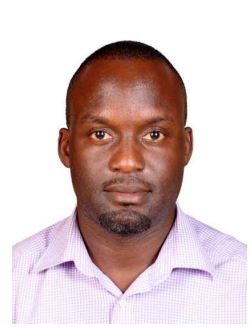

Edwin Mugume (S'12-M'17) received the BSc degree in Electrical Engineering (First Class Honours) from Makerere University, Uganda in 2007, and the MSc degree in Communication Engineering (Distinction) from the University of Manchester, United Kingdom in 2011. He completed the $\mathrm{PhD}$ degree in Electrical and Electronic Engineering from the University of Manchester, UK in 2016.

From 2008 to 2010, he was a radio planning and optimization engineer of cellular networks at Nokia Siemens Networks and Bharti Airtel. He is currently a Lecturer in the Department of Electrical and Computer Engineering, Makerere University. His research interests include energy efficient dense heterogeneous networks, 5G cellular technology, and application of machine learning in wireless networks and systems.

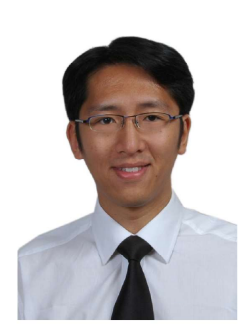

Daniel K. C. So (S'96-M'03-SM'14) received the BEng (Hons) degree in Electrical and Electronics Engineering from the University of Auckland, New Zealand in 1996, and the PhD degree in Electrical and Electronics Engineering from the Hong Kong University of Science and Technology (HKUST) in 2003.

$\mathrm{He}$ joined the University of Manchester as a Lecturer in 2003, and is now a Reader and Discipline Head of Education in the Department of Electrical \& Electronic Engineering. From 1997 to 1998, he was a Senior Software Engineer in Orion Health, New Zealand.

His research interests includes green communications, NOMA, 5G networks, heterogeneous networks, cognitive radio, massive MIMO, D2D communications, cooperative MIMO schemes, multihop communication, channel equalization and estimation techniques. He is currently serving as an Editor of IEEE Transactions on Wireless Communications and IEEE Wireless Communication Letters. He also served as a symposium co-chair of IEEE ICC 2019 and Globecom 2020, and track co-chair for IEEE Vehicular Technology Conference (VTC) Spring 2016, 2017 and 2018 